\title{
Can S100B predict cerebral vasospasms in patients suffering from subarachnoid hemorrhage?
}

\author{
Moshgan Amiri ${ }^{1,2}$, Ramona Astrand $^{1}$ and Bertil Romner ${ }^{1}{ }^{*}$ \\ 1 Department of Neurosurgery, Rigshospitalet, University Hospital of Copenhagen, Copenhagen, Denmark \\ 2 Faculty of Medicine, Copenhagen University, Copenhagen, Denmark
}

\section{Edited by:}

Ronald L. Hayes, Banyan Biomarkers, Inc., USA

\section{Reviewed by:}

Antonino F. Germano, University of Messina, Italy

Firas H. Kobeissy, University of

Florida, USA

\section{${ }^{*}$ Correspondence:}

Bertil Romner, Department of

Neurosurgery, Blegdamsvej 9, 2100

Copenhagen, Denmark

e-mail: bertil.romner@regionh.dk
Background: Protein S100B has proven to be a useful biomarker for cerebral damages. Increased levels of serum and cerebrospinal fluid (CSF) S100B have been shown in patients suffering subarachnoid hemorrhage (SAH), severe head injury and stroke. In patients with $\mathrm{SAH}$, the course of S100B levels has been correlated with neurological deficits and outcome. Cerebral vasospasm is a major contributor to morbidity and mortality. The primary aim of this study was to investigate the potential of S100B protein as a predictor of cerebral vasospasm in patients with severe SAH.

Materials and Methods: Patients with SAH, Fisher grade 3 and 4, were included in the study. Five samples of CSF and serum S100B were collected from each patient. The first sample (baseline sample) was drawn within the first 3 days following ictus and the following four samples, once a day on days $5-8$, with day of ictus defined as day 1 . Clinical suspicion of cerebral vasospasm confirmed by computed tomography angiography was used to diagnose cerebral vasospasm.

Results: A total of 18 patients were included. Five patients (28\%) developed cerebral vasospasm, two $(11 \%)$ developed ventriculitis. There were no significant differences between S100B for those with and without vasospasm. Serum S100B levels in patients with vasospasm were slightly lower within the first 5 days following ictus, compared to patients without vasospasm. Two out of five patients had elevated and increasing serum S100B prior to vasospasm. Only one showed a peak level of S100B 1 day before vasospasm could be diagnosed. Due to the low number of patients in the study, statistical significance could not be reached.

Conclusion: Neither serum nor CSF S100B can be used as predictor of cerebral vasospasm in patients suffering from $\mathrm{SAH}$.

Keywords: protein S100B, subarachnoid hemorrhage, cerebral vasospasm, CT angiography, cerebrospinal fluid, serum

\section{INTRODUCTION}

Subarachnoid hemorrhage (SAH) accounts for approximately 6$8 \%$ of all strokes, and the leading cause is rupture of intracerebral aneurysms in $85 \%$ of the cases. The remaining causes are due to arteriovenous malformation (AVM) bleeding, vertebral artery dissection (about $5 \%$ together), or due to more undefined causes or the perimesencephalic SAH, which account for about $10 \%$ of the cases (van Gijn and Rinkel, 2001). The overall incidence of SAH is approximately 9 per 100,000 persons/year, slightly higher in the Scandinavian countries, an highest in Finland and Japan with 19.7 and 22.7 per 100,000/year, respectively (de Rooij et al., 2007). Mortality and morbidity is high, accounting for up to $50 \%$ in patients suffering from aneurysmal $\mathrm{SAH}$ (aSAH). About $25 \%$ never reach medical attention (Diringer, 2009).

Cerebral vasospasm is an important cause of morbidity and death after SAH (Rowland et al., 2012), for those who survive to receive medical treatment. It is defined as clinical neurological symptoms of ischemia (confusion, decreased level of consciousness, focal neurological deficits), with narrowing of cerebral vessels, visualized by computed tomography angiography (CTA). Cerebral vasospasm occur in approximately one third of aSAH patients (Frontera et al., 2009), and the risk of vasospasm is related to the thickness and amount of blood in the subarachnoid space and/or the presence of intraventricular blood assessed on computed tomography (CT), the Fisher grade (Fisher et al., 1980; Jung et al., 2012). The risk of developing cerebral vasospasm is highest during dag 6-8 following ictus (Weir et al., 1978). SAH patients are clinically monitored with daily neurological examinations, but the means of diagnosing cerebral vasospasm varies. The techniques in use for diagnosing vasospasm are by means of clinical evaluation, transcranial doppler sonography (TCD), CTA, digital subtraction angiography (DSA), or by computed tomography perfusion (CTP) (Yoon et al., 2006; Washington and Zipfel, 2011; Kunze et al., 2012), although the most relevant technique is still not defined (Frontera et al., 2009). 
A biomarker for detection of cerebral vasospasm in patients with SAH, could ideally allow for early detection to prevent delayed ischemic neurological deficits. Protein S100B is a calcium binding protein, predominant in nervous tissue, and mainly expressed in astroglial cells (Donato, 2001). It is also found in extracerebral tissues, such as long bones, fat, melanocytes, heart, and kidneys, though in lesser extent (Anderson et al., 2001; Unden et al., 2005). $\mathrm{S} 100 \mathrm{~B}$ is increased in serum and in cerebrospinal fluid (CSF) after brain injury, mainly as a result of the opening of the blood brain barrier (Marchi et al., 2003). In recent years, studies have shown that $\mathrm{S} 100 \mathrm{~B}$ is useful as a predictive marker for outcome after cerebral infarction (Herrmann and Ehrenreich, 2003; Ahmad et al., 2012), anoxic brain injury (Shinozaki et al., 2009), and SAH (Wiesmann et al., 1997; Stranjalis et al., 2007; Sanchez-Pena et al., 2008).

The aim of our study was to investigate the potential of S100B protein as a predictor of cerebral vasospasm in patients suffering from severe $\mathrm{SAH}$.

\section{MATERIALS AND METHODS PATIENTS}

We prospectively studied patients with $\mathrm{SAH}$, admitted to Copenhagen University Hospital, neurointensive care unit (NICU) between September 2012 and January 2013. The inclusion criteria were: patients with SAH confirmed by CT, Fisher grade 3 and 4 , age 18 years and above, admission and external ventricular drain (EVD) within 3 days of ictus, no other major injuries. Patients who had their EVD removed within the first 8 days after ictus were excluded from the study.

Glasgow Coma Scale (GCS) score (Teasdale and Jennett, 1974) and WFNS grading scale (Teasdale et al., 1988) was used to assess the neurological status on admission. Location and thickness of the hemorrhage on CT scan was determined by the Fisher grading scale (Fisher et al., 1980).

\section{SAMPLES}

A total of five blood samples and five CSF samples were obtained from each patient. The day of ictus was defined as day 1 . The first blood and CSF samples (Baseline sample) were obtained between day 1 and 3, depending on when the patient was transferred to the NICU and had received an EVD. The following four samples were obtained on day 5-8 following ictus. Each blood sample consisted of $4 \mathrm{ml}$ of blood obtained in a SST-tube with separating gel without additives, and the CSF samples consisted of minimum $1 \mathrm{ml}$ of CSF obtained from the EVD. About $4 \mathrm{ml}$ of blood and $1 \mathrm{ml}$ of CSF was taken as waste prior to sampling, as a precaution to contamination and dilution effect. Following collection all samples were centrifuged at $3500 \mathrm{rpm}$ for $7 \mathrm{~min}$ at room temperature. The separated serum and CSF samples were stored at $-80^{\circ} \mathrm{C}$, and thawed prior to analysis. Samples were analyzed with the method of electrochemiluminescence immunoassay, and the equipment used was Elecsys 2010, Modular Analytics E170, Roche Diagnostics (Mannheim, Germany). The lower detection limit was 0.005 and the upper limit $39 \mu \mathrm{g} / \mathrm{L}$.

\section{VASOSPASM}

Daily neurological status was assessed to determine clinical signs of worsening with cerebral vasospasm as the primary cause. Cerebral vasospasm was confirmed by CTA.

\section{STATISTICS}

Statistical analysis was performed in IBM SPSS Statistics ver. 19.0.0. Figures and tables were computed in Microsoft Excel 2007. Student $t$-test was performed for comparing mean S100B levels between patients with and without cerebral vasospasm. Significance level was set to 0.05 .

\section{ETHICAL CONSIDERATIONS}

The study and the collection of S100B in both serum and CSF had been approved by the local ethics committee.

\section{RESULTS}

A total of 18 patients with severe SAH were included, 16 had an aSAH, one had SAH due to a small AVM bleeding, and one due to dissection of the vertebral artery. Mean age was 60 years (range 42-84 years), there were 11 females and 7 males. The mean GCS score on admission was 8 , the mean score of the WFNS grading scale was 3.5, and the mean Fisher grade was 3.6. Patients with aSAH were treated with endovascular coiling (81\%) or surgical clipping (19\%). The two patients without aSAH were treated conservatively.

The baseline samples were obtained on day 1 from one patient, on day 2 from four patients and on day 3 from 13 patients. Five patients developed cerebral vasospasm during the first week. None developed cerebral vasospasm later than on day 8 . Three patients died, of which one developed cerebral vasospasm during the trial period. Two patients developed bacterial ventriculitis during the trial period.

When comparing patients who developed cerebral vasospasm with those who did not, there were no significant differences in GCS score at admission, Fisher grade or WFNS grade. Mean age was slightly, but not significantly, higher in patients that developed cerebral vasospasm (Table 1). The mean serum S100B level in

Table 1 | Characteristics of patients with cerebral vasospasm compared to patients without.

\begin{tabular}{|c|c|c|}
\hline & $\begin{array}{l}\text { Cerebral } \\
\text { vasospasm } \\
n=5\end{array}$ & $\begin{array}{l}\text { No cerebral } \\
\text { vasospasm } \\
n=13\end{array}$ \\
\hline Gender (M:F) & $2: 3$ & $5: 8$ \\
\hline Mean age (years) & 67 & 58 \\
\hline Mean GCS at admission & 8 & 9 \\
\hline Mean WFNS grading score at admission & 3.6 & 3.5 \\
\hline Mean Fisher grading score & 3.6 & 3.5 \\
\hline Mean body temperature $\left({ }^{\circ} \mathrm{C}\right)$ & 38.0 & 38.0 \\
\hline Intracranial infection & 0 & 2 \\
\hline Death & 1 & 2 \\
\hline \multicolumn{3}{|l|}{ Sample collection $(n)$} \\
\hline \multicolumn{3}{|l|}{ Baseline } \\
\hline Day 1 & 1 & 0 \\
\hline Day 2 & 1 & 3 \\
\hline Day 3 & 3 & 10 \\
\hline Days 5-8 & 5 & 13 \\
\hline
\end{tabular}

GCS, glasgow coma scale; WFNS, World federation of neurological societies grading scale. 
patients who developed cerebral vasospasm compared to those who did not, was lower within the first 5 days after ictus. Following day 5, serum S100B levels in the group with cerebral vasospasm, was increased and exceeded the mean level of serum S100B in patients who did not developed vasospasm, though not statistically significant (Table 2). The mean CSF S100B levels in patients who developed cerebral vasospasm were lower compared to patients without vasospasm, but not statistically significant. The peak and mean body temperatures were generally high, but did not differ between those who developed vasospasm and those who did not.

Among the five patients who developed cerebral vasospasm, baseline samples of serum and CSF S100B were drawn on day 1 in one patient, at day 2 in one patient, and at day 3 in three. Two patients had increasing levels of serum S100B compared to the other three, which had an overall decreasing tendency and normal levels of serum S100B when vasospasm was diagnosed.
Only one patient reached a peak level of serum S100B the day before vasospasm was confirmed (Figure 1, patient B), while the other patient reached peak S100B 1 day after angiographic confirmation of cerebral vasospasm and ongoing cerebral infarction (Figure 1, patient E). Both patients had a WFNS score of 5 and a GCS of 3 at admission, and both developed pneumonia during the trial period. The other three patients with cerebral vasospasm had slightly lower WFNS and slightly higher GCS scores at admission, and none had pneumonia or other infections diagnosed during the trial period.

Among patients who did not developed cerebral vasospasm, the baseline samples were drawn on day 2 in three patients and on day 3 in 13 patients. Two developed bacterial ventriculitis, with a sudden 10-fold increase in serum S100B levels. One reached peak level of serum S100B the day before developing ventriculitis, while peak levels for the other patient occurred on the same day as the

Table 2 | Mean serum and CSF S100B levels in patients with cerebral vasospasm compared to patients without*.

\begin{tabular}{|c|c|c|c|c|}
\hline \multirow[t]{2}{*}{ Samples } & \multicolumn{2}{|c|}{ Mean serum S100B $(\mu \mathrm{g} / \mathrm{L})$} & \multicolumn{2}{|c|}{ Mean CSF S100B $(\mu \mathrm{g} / \mathrm{L})$} \\
\hline & $\begin{array}{l}\text { Cerebral } \\
\text { vasospasm } N=5\end{array}$ & $\begin{array}{l}\text { No cerebral } \\
\text { vasospasm } N=13\end{array}$ & $\begin{array}{l}\text { Cerebral } \\
\text { vasospasm } N=5\end{array}$ & $\begin{array}{l}\text { No cerebral } \\
\text { vasospasm } N=13\end{array}$ \\
\hline Baseline & 0.10 & 0.19 & 23.5 & 29.6 \\
\hline Day 5 & 0.15 & 0.37 & 13.8 & 21.8 \\
\hline Day 6 & 0.21 & 0.15 & 10.1 & 16.3 \\
\hline
\end{tabular}

*There are no statistical differences between the two groups (vasospasm vs. no vasospasm).

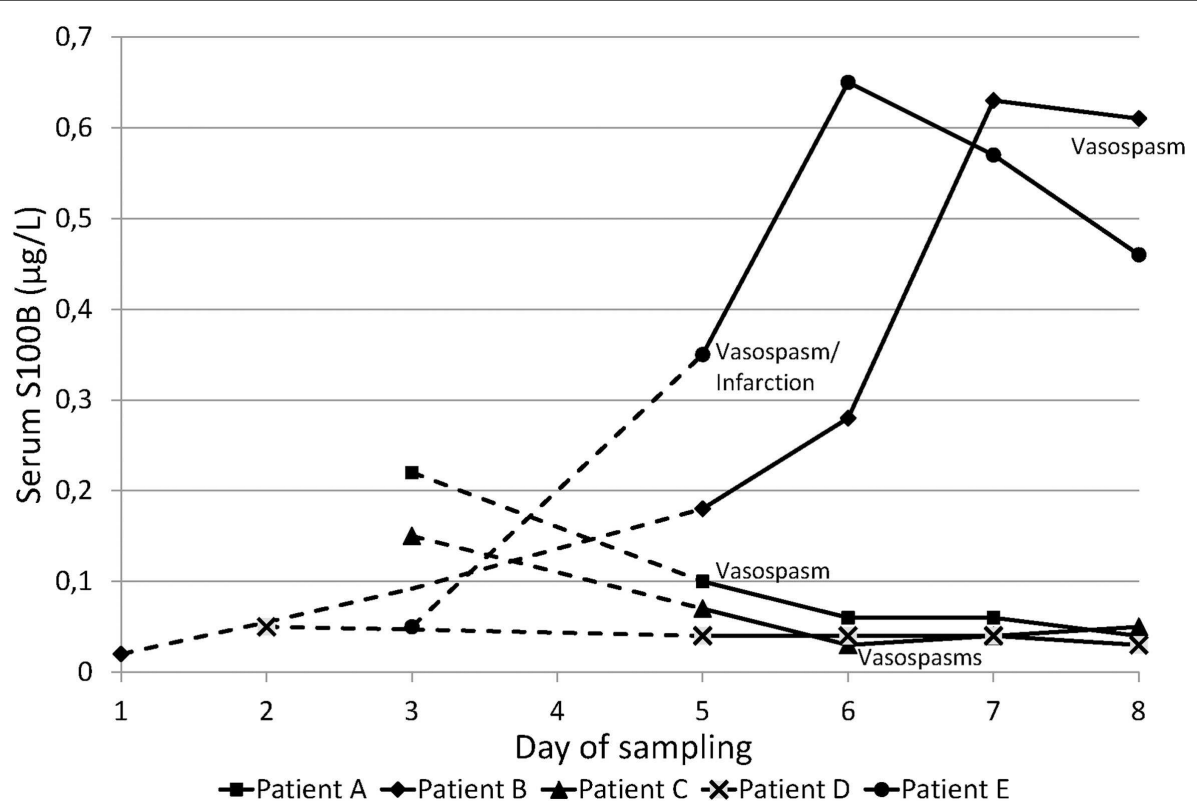

FIGURE 1 | Serum S100B levels for the five patients with cerebral vasospasm. Day $1=$ day of ictus. Baseline sampling was made between day 1 and 3. The dotted lines are extrapolations from baseline sample to the second sample drawn on day 5 . The day on which cerebral vasospasm was confirmed is indicated for each patient. 
infection was diagnosed. No such relation was observed for CSF S100B.

\section{DISCUSSION}

We studied a total of $18 \mathrm{SAH}$ patients by measuring S100B in serum and CSF within the first 8 days following ictus. Five patients developed cerebral vasospasm, detected by neurological deterioration and confirmed with CTA. No significant differences for S100B levels were observed when comparing patients who developed cerebral vasospasm to patients who did not, and S100B failed predict the development of cerebral vasospasm.

Only two patients with cerebral vasospasm developed increased levels of serum S100B during the trial period. Both patients were in worse neurological condition (GCS 3, WFNS 5 at admission) compared to the other patients who developed cerebral vasospasm (Stranjalis et al., 2007). In addition, these two patients developed pneumonia during the trial period. Two patients in the group without cerebral vasospasm developed ventriculitis. High levels of serum S100B were found on the day before or on the same day as the clinical diagnosis of bacterial ventriculitis was set. The results indicate an association between increased serum S100B and intracerebral infections as shown in a previous study (Unden et al., 2004).

Measurements of S100B in our patients were done once daily with baseline sample obtained within the first 3 days of ictus and the rest of the four samples on days 5-8 following ictus. The baseline samples were obtained within the first 3 days of ictus, as cerebral vasospasm usually do not occur before day 4 following ictus. As the highest risk for developing vasospasm is between 6 and 8 days following the subarachnoid bleeding (Weir et al., 1978), the subsequent samples were drawn on day 5-8. None of the patients developed either clinical or angiographic vasospasm after day 8.

In two patients the bleeding source was not an aneurysm, but a small deep AVM in one patient, and a vertebral artery dissection in the other. The risk of developing cerebral vasospasm increases with increasing volume of subarachnoid blood visualized by CT (Fisher et al., 1980; Adams et al., 1987). Both of these patients had high amount of subarachnoid blood visualized on CT (Fisher grade 3 and 4), and thus high risk of developing cerebral vasospasm.

Currently, neurosurgical centers use daily assessment of neurological status, TCD sonography and CTA to determine the development of cerebral vasospasm. Most patients suffering from severe SAH, have already, prior to transport to the NICU, been sedated and intubated, making the assessment of the neurological

\section{REFERENCES}

Adams, H. P. Jr., Kassell, N. F., Torner, J. C., and Haley, E. C. Jr. (1987). Predicting cerebral ischemia after aneurysmal subarachnoid hemorrhage: influences of clinical condition, CT results, and antifibrinolytic therapy. A report of the Cooperative Aneurysm Study. Neurology 37, 1586-1591. doi:10.1212/WNL.37.10.1586

Ahmad, O., Wardlaw, J., and Whiteley, W. N. (2012). Correlation of levels of neuronal and glial markers with radiological measures of infarct volume in ischaemic stroke: a systematic review. Cerebrovasc. Dis. 33, 47-54. doi:10.1159/000332810

Anderson, R. E., Hansson, L. O., Nilsson, O., Dijlai-Merzoug, R., and Settergren, G. (2001). High serum S100B levels for trauma patients without head injuries. $\mathrm{Neu}$ rosurgery 48, 1255-1258; discussion 1258-1260. doi:10.1097/00006123200106000-00012

status in these patients challenging. In our study, clinical suspicion of vasospasm in addition with CTA, rather than TCD, was used to diagnose cerebral vasospasm. CTA has been found to correlate well (overall agreement of 95\%) with DSA (Yoon et al., 2006), which has been considered as the gold standard in detecting cerebral vasospasm. Furthermore, Kunze et al. (2012) have shown that the accuracy of neurological examination and CTP is higher in detecting cerebral vasospasm than TCD, and in a systematic review by Lysakowski and colleagues, they concluded that although TCD has a high specificity $(0.99)$ and positive predictive value $(0.97)$ for detecting vasospasm, this accounts only for the middle cerebral artery. For all the other arteries, there is no evidence for the usefulness of TCD as a diagnostic tool for vasospasm (Lysakowski et al., 2001).

Our study also show a tendency toward lower serum S100B levels during the first 5 days following $\mathrm{SAH}$ in patients who developed cerebral vasospasm compared to those who did not. These results were not statistically significant, thus not supporting useful value of serum S100B in predicting vasospasm. Similar results have been found by Oertel et al. (2006) who showed lower levels of serum S100B within the first 3 days after SAH in patients who developed cerebral vasospasm compared to those who did not. Cerebral vasospasm was determined by neurological deterioration and increasing flow velocity on TCD.

Identifying a specific biomarker for prediction of cerebral vasospasm in this group of patients is of high value, since earlier detection and hence earlier treatment of vasospasm could lower the morbidity and mortality in this group of patients. The statistical analysis of our end results are limited by the small number of cases enrolled in the study. We can, however, conclude that, although S100B is a promising prognostic biomarker of secondary brain damage and outcome in patients with SAH (Stranjalis et al., 2007; Sanchez-Pena et al., 2008), the potential of $\mathrm{S} 100 \mathrm{~B}$ as a predictor of cerebral vasospasm is very limited. This is in accordance with two previous studies (Moritz et al., 2010; Jung et al., 2013). The need of a marker in predicting cerebral vasospasm still remains, and other biomarkers such as myeloperoxidase (Lim et al., 2012), amino acids in addition to microdialysis (Jung et al., 2013), endothelin-1, interleukin-6, and indicators of thrombin activity might be of greater utility (Lad et al., 2012).

\section{ACKNOWLEDGMENTS}

The authors thank nurse Pia Breum Ferdinand, who assisted with recruitment and sampling.

de Rooij, N. K., Linn, F. H., van der Plas, J. A., Algra, A., and Rinkel, G. J. (2007). Incidence of subarachnoid haemorrhage: a systematic review with emphasis on region, age, gender and time trends. J. Neurol. Neurosurg. Psychiatr. 78, 1365-1372. doi:10.1136/jnnp.2007.117655

Diringer, M. N. (2009). Management of aneurysmal subarachnoid hemorrhage. Crit. Care Med. 37, 432-440. doi:10.1097/CCM.0b013e318195 $865 \mathrm{a}$
Donato, R. (2001). S100: a multigenic family of calcium-modulated proteins of the EF-hand type with intracellular and extracellular functional roles. Int. J. Biochem. Cell Biol. 33, 637-668. doi:10.1016/S13572725(01)00046-2

Fisher, C. M., Kistler, J. P., and Davis, J. M. (1980). Relation of cerebral vasospasm to subarachnoid hemorrhage visualized by computerized tomographic scanning. Neurosurgery 6, 1-9. 
doi:10.1227/00006123-19800100000001

Frontera, J. A., Fernandez, A., Schmidt, J. M., Claassen, J., Wartenberg, K. E., Badjatia, N., et al. (2009). Defining vasospasm after subarachnoid hemorrhage: what is the most clinically relevant definition? Stroke 40, 1963-1968. doi:10.1161/STROKEAHA.108.54 4700

Herrmann, M., and Ehrenreich, H. (2003). Brain derived proteins as markers of acute stroke: their relation to pathophysiology, outcome prediction and neuroprotective drug monitoring. Restor. Neurol. Neurosci. 21, 177-190.

Jung, C. S., Lange, B., Zimmermann, M., and Seifert, V. (2013). CSF and serum biomarkers focusing on cerebral vasospasm and ischemia after subarachnoid hemorrhage. Stroke Res. Treat. 2013, 560305. doi:10.1155/2013/560305

Jung, S. W., Lee, C. Y., and Yim, M. B. (2012). The relationship between subarachnoid hemorrhage volume and development of cerebral vasospasm. J. Cerebrovasc. Endovasc. Neurosurg. 14, 186-191. doi:10.7461/jcen.2012.14.3.186

Kunze, E., Pham, M., Raslan, F., Stetter, C., Lee, J. Y., Solymosi, L., et al. (2012). Value of perfusion $\mathrm{CT}$, transcranial doppler sonography, and neurological examination to detect delayed vasospasm after aneurysmal subarachnoid hemorrhage. Radiol. Res. Pract. 2012, 231206. doi:10.1155/2012/231206

Lad, S. P., Hegen, H., Gupta, G., Deisenhammer, F., and Steinberg, G. K. (2012). Proteomic biomarker discovery in cerebrospinal fluid for cerebral vasospasm following subarachnoid hemorrhage. $J$. Stroke Cerebrovasc. Dis. 21, 30-41. doi:10.1016/j.jstrokecerebrovasdis. 2010.04.004

Lim, M., Bower, R. S., Wang, Y., Sims, L., Bower, M. R., Camara-Quintana,
J., et al. (2012). The predictive value of serum myeloperoxidase for vasospasm in patients with aneurysmal subarachnoid hemorrhage. Neurosurg. Rev. 35, 413-419; discussion 419. doi:10.1007/s10143012-0375-4

Lysakowski, C., Walder, B., Costanza, M. C., and Tramer, M. R. (2001). Transcranial doppler versus angiography in patients with vasospasm due to a ruptured cerebral aneurysm: a systematic review. Stroke 32, 2292-2298. doi:10.1161/hs1001.097108

Marchi, N., Rasmussen, P., Kapural, M., Fazio, V., Kight, K., Mayberg, M. R., et al. (2003). Peripheral markers of brain damage and blood-brain barrier dysfunction. Restor. Neurol. Neurosci. 21, 109-121.

Moritz, S., Warnat, J., Bele, S., Graf, B. M., and Woertgen, C. (2010). The prognostic value of NSE and S100B from serum and cerebrospinal fluid in patients with spontaneous subarachnoid hemorrhage. J. Neurosurg. Anesthesiol. 22, 21-31. doi:10. 1097/ANA.0b013e3181bdf50d

Oertel, M., Schumacher, U., McArthur, D. L., Kastner, S., and Boker, D. K. (2006). S-100B and NSE: markers of initial impact of subarachnoid haemorrhage and their relation to vasospasm and outcome. J. Clin. Neurosci. 13, 834-840. doi:10.1016/j.jocn.2005.11.030

Rowland, M. J., Hadjipavlou, G., Kelly, M., Westbrook, J., and Pattinson, K. T. (2012). Delayed cerebral ischaemia after subarachnoid haemorrhage: looking beyond vasospasm. Br. J. Anaesth. 109, 315-329. doi:10.1093/bja/aes264

Sanchez-Pena, P., Pereira, A. R., Sourour, N. A., Biondi, A., Lejean, L., Colonne, C., et al. (2008). $\mathrm{S} 100 \mathrm{~B}$ as an additional prognostic marker in subarachnoid aneurysmal hemorrhage. Crit. Care Med. 36, 2267-2273. doi:10. 1097/CCM.0b013e3181809750
Shinozaki, K., Oda, S., Sadahiro, T. Nakamura, M., Abe, R., Nakada, T. A., et al. (2009). Serum S-100B is superior to neuron-specific enolase as an early prognostic biomarker for neurological outcome following cardiopulmonary resuscitation. Resuscitation 80, 870-875. doi:10. 1016/j.resuscitation.2009.05.005

Stranjalis, G., Korfias, S., Psachoulia, C., Kouyialis, A., Sakas, D. E., and Mendelow, A. D. (2007). The prognostic value of serum S-100B protein in spontaneous subarachnoid haemorrhage. Acta Neurochir. (Wien) 149, 231-237; discussion 237-238. doi:10.1007/s00701-006-1106-9

Teasdale, G., and Jennett, B. (1974). Assessment of coma and impaired consciousness. A practical scale. Lancet 2, 81-84. doi:10.1016/S01406736(74)91639-0

Teasdale, G. M., Drake, C. G., Hunt, W., Kassell, N., Sano, K., Pertuiset, B., et al. (1988). A universal subarachnoid hemorrhage scale: report of a committee of the World Federation of Neurosurgical Societies. J. Neurol. Neurosurg. Psychiatr. 51, 1457. doi:10.1136/jnnp.51.11.1457

Unden, J., Bellner, J., Eneroth, M. Alling, C., Ingebrigtsen, T., and Romner, B. (2005). Raised serum S100B levels after acute bone fractures without cerebral injury. J. Trauma 58, 59-61. doi:10. 1097/01.TA.0000130613.35877.75

Unden, J., Christensson, B., Bellner, J., Alling, C., and Romner, B. (2004). Serum S100B levels in patients with cerebral and extracerebral infectious disease. Scand. J. Infect. Dis. 36, 10-13. doi:10.1080/00365540310017294

van Gijn, J., and Rinkel, G. J. (2001). Subarachnoid haemorrhage: diagnosis, causes and management. Brain 124, 249-278. doi:10.1093/brain/124.2.249

Washington, C. W., and Zipfel, G. J. (2011). Detection and monitoring of vasospasm and delayed cerebral ischemia: a review and assessment of the literature. Neurocrit. Care 15, 312-317. doi:10.1007/s12028011-9594-8

Weir, B., Grace, M., Hansen, J., and Rothberg, C. (1978). Time course of vasospasm in man. J. Neurosurg. 48, 173-178. doi:10.3171/jns.1978.48.2.0173

Wiesmann, M., Missler, U., Hagenstrom, H., and Gottmann, D. (1997). S-100 protein plasma levels after aneurysmal subarachnoid haemorrhage. Acta Neurochir. (Wien) 139, 1155-1160. doi:10.1007/BF01410976

Yoon, D. Y., Choi, C. S., Kim, K. H., and Cho, B. M. (2006). Multidetectorrow CT angiography of cerebral vasospasm after aneurysmal subarachnoid hemorrhage: comparison of volume-rendered images and digital subtraction angiography. AJNR Am. J. Neuroradiol. 27, 370-377.

Conflict of Interest Statement: The authors declare that the research was conducted in the absence of any commercial or financial relationships that could be construed as a potential conflict of interest.

Received: 22 February 2013; accepted: 21 May 2013; published online: 06 June 2013.

Citation: Amiri M, Astrand $R$ and Romner B (2013) Can $S 100 B$ predict cerebral vasospasms in patients suffering from subarachnoid hemorrhage? Front. Neurol. 4:65. doi: 10.3389/fneur.2013.00065 This article was submitted to Frontiers in Neurotrauma, a specialty of Frontiers in Neurology.

Copyright (C) 2013 Amiri, Astrand and Romner. This is an open-access article distributed under the terms of the Creative Commons Attribution License, which permits use, distribution and reproduction in other forums, provided the original authors and source are credited and subject to any copyright notices concerning any third-party graphics etc. 